\title{
Diacronie
}

Studi di Storia Contemporanea

$N^{\circ} 30,2$ | 2017

Ponti fra nazioni e continenti

\section{Storia del contributo italiano alla viticoltura sudafricana dalle origini alla contemporaneità}

\section{Claudio Sessa}

\section{(2) OpenEdition}

\section{Journals}

\section{Edizione digitale}

URL: http://journals.openedition.org/diacronie/5590

DOI: 10.4000/diacronie.5590

ISSN: 2038-0925

\section{Editore}

Association culturelle Diacronie

\section{Notizia bibliografica digitale}

Claudio Sessa, «Storia del contributo italiano alla viticoltura sudafricana dalle origini alla contemporaneità », Diacronie [Online], №30, 2 | 2017, documento 1, Messo online il 29 juillet 2017, consultato il 30 avril 2019. URL : http://journals.openedition.org/diacronie/5590 ; DOI : 10.4000/ diacronie. 5590 


\section{Diacronie}

Studi di Storia Contemporanea

30, 2/2017

Ponti fra nazioni e continenti: diplomazia, immaginari e conoscenze tecniche

\section{Storia del contributo italiano alla viticoltura sudafricana dalle origini alla contemporaneità}

Claudio SESSA

Per citare questo articolo:

SESSA, Claudio, «Storia del contributo italiano alla viticoltura sudafricana dalle origini alla contemporaneità», Diacronie. Studi di Storia Contemporanea : Ponti fra nazioni e continenti: diplomazia, immaginari e conoscenze tecniche, 30, 2/2017, 29/7/2017,

URL: < http://www.studistorici.com/2017/07/29/sessa_numero_30/ >

Diacronie Studi di Storia Contemporanea $\rightarrow$ http://www.diacronie.it Rivista storica online. Uscita trimestrale.

redazione.diacronie@hotmail.it

Comitato di direzione: Naor Ben-Yehoyada - João Fábio Bertonha - Christopher Denis-Delacour - Maximiliano Fuentes Codera Anders Granås Kjøstvedt - John Paul Newman - Deborah Paci - Niccolò Pianciola - Spyridon Ploumidis - Wilko Graf Von Hardenberg

Comitato di redazione: Jacopo Bassi - Luca Bufarale - Gianluca Canè - Fausto Pietrancosta - Alessandro Salvador - Matteo Tomasoni Diritti: gli articoli di Diacronie. Studi di Storia Contemporanea sono pubblicati sotto licenza Creative Commons 3.0. Possono essere

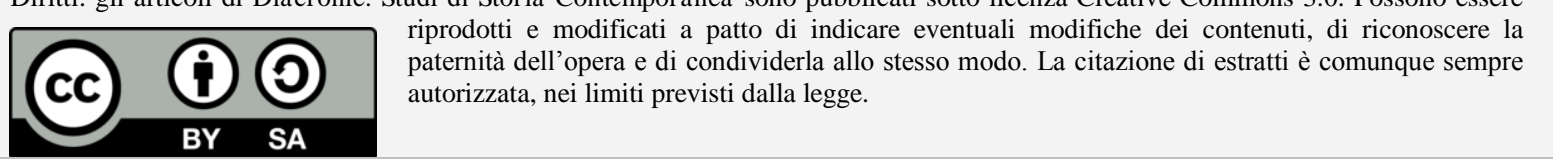




\title{
1/ Storia del contributo italiano alla viticoltura sudafricana dalle origini alla contemporaneità
}

\author{
Claudio SESSA
}

Gli italiani nel tempo hanno stretto un rapporto simbiotico con la pianta della vite, con il suo frutto, l'uva e con il suo prodotto, il vino. Hanno esportato nel mondo le abilità e le qualità artigianali, il know how di conoscenze utili allo sviluppo dell'industria viti-vinicola in qualsiasi luogo o regione del mondo, come in Sudafrica. La grande abilità maturata nei secoli ha rappresentato un utile strumento, un "biglietto da visita" con cui potersi inserire in società e contesti differenti da quello nazionale, mediante un processo di integrazione e confronto che ha concorso alla modificazione ed all'evoluzione del settore viti-vinicolo e dei costumi alimentari sudafricani. Il presente articolo mira a ricostruire la storia enologica sudafricana mediante l'analisi del contributo italiano allo sviluppo del settore stesso in una prospettiva di lungo termine che va dal XVII sec. fino alla contemporaneità approfondendo episodi trascurati ed esenti da trattazione scientifico-accademiche.

\section{Premessa}

Negli ultimi decenni il comparto viti-vinicolo sudafricano ha fatto registrare una crescita quantitativa e qualitativa senza precedenti affermandosi a livello mondiale tra i più rinomati mercati vinicoli. Dietro questa ascesa si cela una storia molto complessa ${ }^{1}$ e dalle radici antiche che coinvolge, in parte, anche gli italiani. Il presente contributo vuole ricostruire la storia della presenza e del contributo italiano nel settore viti-vinicolo sudafricano focalizzando l'attenzione maggiormente su quelle esperienze "innovative" che hanno segnato in modo indelebile il destino del vino e della storia enologica locale ${ }^{2}$.

\footnotetext{
${ }^{1}$ Cfr. FOURIE, Johan, VON FINTEL, Dieter, The Fruit of the Vine? An Augmented Endowments-Inequality Hypothesis and the Rise of an Elite in the Cape Colony, Helsinki, UNU-WIDER, WIDER Working Paper, 112, 2010, pp. 1-29.

${ }^{2}$ Riguardo alla storia degli italiani in Sudafrica si veda: SANI, Gabriele, Storia degli italiani in Sudafrica 14891989, Edenvale, Zonderwater Block, 1989; per un primo tentativo di ricostruzione dei flussi migratori italiani in Sudafrica si vedano anche: IACOPONI, Valentina, Campi d'oro e strade di ferro, il Sudafrica e l'immigrazione italiana tra ottocento e novecento, Roma, XL Edizioni, 2013; MACIOTI, Maria Immacolata, ZACCAI, Claudia, Italiani in Sudafrica, le trasformazioni culturali della migrazione, Milano, Guerini scientifica, 2006; GIULIANI-
} 


\section{Le origini: la Compagnia delle Indie Orientali Olandese (VOC)}

Le origini della viticoltura sudafricana sono strettamente intrecciate con la storia della presenza della VOC, la Compagnia delle Indie Orientali Olandese ${ }^{3}$, presso il Capo di Buona Speranza e del progetto di trasformazione di quest'ultimo in stazione di rifornimento e di assistenza in grado di fornire approvvigionamenti ai convogli marittimi impegnati nel commercio con le Indie. A questo scopo, sotto le pressioni del segretario della Compagnia, Van Dam, il Consiglio dei Diciassette, decise di prendere in considerazione la proposta di fondare una roccaforte alla punta meridionale del continente africano qualora ci fossero state le condizioni ${ }^{4}$. Già i portoghesi, con la scoperta di Bartolomeo Diaz nel 1487, avevano beneficiato di tale sito come scalo commerciale per i loro traffici con l' Oriente e successivamente gli olandesi designarono Jan Van Riebeeck ${ }^{5}$ quale capitano di una spedizione con l'incarico di costruire una stazione di scalo presso il Capo di Buona Speranza. Egli approdò sulle coste dell'odierno Sudafrica nel $1652^{6}$ costruendo dapprima un forte, poi selezionando un sito per le attività di giardinaggio ed infine del terreno utile alla semina. Dopo aver stabilito un punto d'approdo sicuro ed uno scalo utile ai marinai, il Governatore Van Riebeeck iniziò a cercare una soluzione che limitasse l'incidenza dello scorbuto tra gli equipaggi e che potesse sostituire l'acqua come bevanda di bordo. All'epoca tutti i capitani di vascello portavano con sé una copia del testo di un certo

BALESTRINO, Maria Clotilde, Gli italiani nel Sudafrica, Napoli, Geocart Editrice Sas, 1995; BINI, Adolfo Giuseppe, Italiani in Sudafrica, Milano, Scuola Arti Grafiche Gianelli, 1957; OTTAVIANO, Chiara, Italians in South Africa, in L'emigrazione italiana, 1870-1970. Atti dei colloqui di Roma, vol. II, Roma, Ministero per i beni e le attività culturali, Direzione generale per gli archivi, 2002; NATILI, Daniele, Una parabola migratoria. Fisionomie e percorsi delle collettività italiane in Africa, Viterbo, Sette Città, 2009.

In merito al contributo degli italiani allo sviluppo del Sudafrica cfr. FERREIRA, Ilse, Sulle orme degli italiani in Sudafrica, Kruger National Park, Jacana Media, 2009.

${ }^{3}$ Il nome ufficiale era: Vereenigde Nedeslantsche Geoctroyeer Oostindische Compagnie.

${ }^{4} \mathrm{Nel} 1645$ venne costruito un approdo nella regione del Capo ma non ebbe successo. Solo nel 1647, a seguito del naufragio della Nieuw Haarlem, avvenuto il 25 marzo del 1647 nella Baia della Tavola, gli olandesi cominciarono a valutare la possibilità di installare un approdo sicuro nella regione. Infatti, a seguito dell'esperienza di sessanta coloni naufragati sulle coste del Sud Africa e sopravvissuti per un anno, commerciando e stabilendo rapporti con gli indigeni, fu chiaro che le possibilità di insediamento erano concrete. Il 26 luglio del 1949 Janszen e Matthys Proot presentarono il rapporto al Consiglio dei Diciassette nel quale veniva paventata la reale possibilità di costruire installazioni permanenti sotto il controllo della sede regionale della Compagnia, situata a Batavia in Insulindia. Per saperne di più cfr. LUGAN, Bernard, Storia del Sudafrica dall'antichità ad oggi, Milano, Garzanti, 1989.

${ }^{5}$ Johan Anthoniszoon van Riebeeck ( 21 aprile 1619- 21 gennaio 1677) è stato un esploratore olandese, amministratore di colonie olandese ed è stato il fondatore di Città del Capo e del settore enologico sudafricano.

${ }^{6}$ Partirono dall'Olanda il 25 dicembre del 1651 a bordo di cinque navi dirette verso il Sud Africa: Dromaderis, Goede Hoop, Reiger, Wallis e Oliphant. Il comandante era Jan Van Riebeeck che portò con se la moglie Maria de la Queillerie ed il figlio di quattro anni. Per saperne di più cfr. LUGAN, Bernard, op. cit. 
Conrad Knuthman dal titolo Medulla Destillatoria et Medica ${ }^{7}$ dove nel capitolo iniziale venivano descritte le proprietà naturali del vino nel trattamento dello scorbuto e nell'apporto di vitamine. La Compagnia così ordinò a tutte le imbarcazioni di caricare sui vascelli grandi quantità di vino ma sfortunatamente la bevanda, di bassa qualità, risultava inservibile ancor prima di raggiungere l'equatore.

Il Governatore allora decise di impiantare delle viti nella regione del Capo. Nel 1654 il primo carico di viti (con Muscat, Green Grape, Muscadel and French Grape) e di esperti viticoltori approdò alla punta meridionale del continente africano. Dopo le prime difficoltà dettate dall'inesperienza e dalla dura lotta contro la natura, il 2 febbraio del 1659, data simbolo nella storia del vino sudafricano, il Governatore Van Riebeeck annotava nel suo diario:

Today - God be praise - wine press for the first time from the Cape grapes, and from the virgin must, fresh from the coop, a salpe take [...] prende from the three young vines that have been growing here for two years [...] yielding 12 mengles must (15 litres) from French or Muscadel Grapes, the Hanepoot Spanish not yet ripe ${ }^{8}$.

I coloni iniziarono a fondare nuove fattorie ed incrementarono il terreno per la coltivazione della vite ma le resistenze dei popoli indigeni, tra cui i Khoi-san, rallentarono l'espansione territoriale dei free burgers (liberi cittadini) e della compagnia olandese. Solamente con l'elezione e l'arrivo del Governatore Simon Van der Stel ${ }^{9}$ ebbe inizio un progetto di espansione strutturato consistente nella fondazione di diversi centri nella regione del Capo, tra cui Stellenbosch nel 1683 e Paarl nel 1687, nei decenni successivi centri principali della produzione vinicola.

Il nuovo Governatore, Van der Stel, (raggiunse la carica di Governatore a circa quarant'anni il 12 ottobre del 1679) era un uomo di grande cultura, educato nelle migliori accademie olandesi e dotato di una grande passione per l'agricoltura, in particolare per i vini; per la coltivazione di quest'ultimo si avvalse della consulenza di un francese, il cui nome era Jean Marieau, esperto viticoltore con esperienza nei vitigni del sud della Francia ${ }^{10}$. "L'industria vinicola" sudafricana conseguì i primi successi sia dal punto di vista quantitativo che qualitativo, dimostrando l'importanza delle intuizioni del Governatore riguardo la pigiatura di uve mature e la salubrità

\footnotetext{
${ }^{7}$ LEIPOLDT, Louis Christian Frederik, 300 years of Cape wine, London-Cape Town-Johannesburg, TafelbergUitgewers Beperk-Nasionale Boekhandel (Publishers) Ltd., 1952, p. 3.

${ }^{8}$ Ibidem, p. 4.

${ }^{9}$ Simon Van der Stel (1 giugno 1691 - 2 novembre 1699) fu comandante e governatore della Colonia del Capo. Espanse i territori controllati dal governo olandese e fondò diversi centri; favorì lo sviluppo delle attività agricole mediante la fondazione di fattorie (farms) e la coltivazione della vite, che sarebbe diventato un elemento fondamentale della vita della colonia. $\mathrm{Fu}$ il fondatore di Constantia, un tenuta in cui mise a coltura diversi prodotti tra cui il vino.

${ }^{10}$ NOBLE, John, South Africa, Past and Present- Short History of European Settlements at the Cape, London, Longmans \& Co., 1877.
} 
della cantina dove far fermentare il vino. La sua tenuta, chiamata Constantia ${ }^{11}$ fu il centro della sperimentazione e della produzione vinicola, basata sulla diversità e sulla varietà dei vitigni. Il vino prodotto nella tenuta del Governatore conquistò una reputazione di eccellenza nella regione del Capo ma anche a livello internazionale: «the wine from Constantia is of a much higher quality than any sent out so far» ${ }^{12}$. Il viaggiatore francese Francois Valentijn visitò Constantia ed annotò nel suo resoconto di viaggio: «also that until name there is no wine to be compared to the red Constantia wine» ${ }^{13}$.

Alla fine del secolo, a seguito della formazione di piccoli latifondi, furono piantate più di un milione e mezzo di viti, così diceva Van der Stel: «On account of the vine fluorishing here so well, many persons are incline to neglect other farming and to plant large vineyards ${ }^{14}$. Inoltre il Governatore iniziò ad incoraggiare la diversificazione del prodotto, spronando i latifondisti ad utilizzare diversi vitigni per incrementare ulteriormente la qualità del prodotto ancora troppo «exceptionally harsh $»^{15}$. Incominciò anche l'esportazione verso Batavia e l'olanda, dove i il Consiglio dei Diciassette lo giudicò «not bad» ${ }^{16}$ ma troppo costoso e meno competitivo di quello Spagnolo.

Per migliorare ulteriormente la produzione e la qualità del vino gli olandesi pensarono bene di sfruttare a proprio vantaggio un evento politico di grande rilevanza che scosse l'Europa, la revoca dell'Editto di Nantes, il 18 ottobre del 1685, con il quale Luigi XIV proibiva il culto evangelico in tutto il Regno di Francia.

\section{Ugonotti e Valdesi: le trattative con il Consiglio dei Diciassette ed il popolamento della Colonia del Capo}

La revoca dell'Editto di Nantes ebbe conseguenze importanti sugli equilibri socio-politici degli Stati Europei e sui relativi possedimenti coloniali. All'indomani della decisione del Re di Francia di limitare nuovamente la libertà religiosa, la popolazione francese di origine Ugonotta fu costretta ad intraprendere la strada dell'esilio, muovendo verso territori occupati da propri correligionari: Germania, Svizzera, Olanda ed il Piemonte savoiardo.

Luigi XVI stizzito dalla posizione accondiscendente di quest'ultimo Stato, esortò il Duca Vittorio Amedeo II di Savoia ad emulare la decisione transalpina così da poter estirpare, a sua

\footnotetext{
${ }^{11}$ Constantia è il nome dato alla tenuta fondata da Simon Van der Stel nel 1685.

${ }^{12}$ JAMES, Tim, Wines of the new South Africa- tradition and revolution, Berkeley-Los Angeles-London, University of California Press, 2013, p. 26.

${ }^{13}$ Ibidem.

${ }^{14}$ LEIPOLDT, Louis Christian Frederik, op. cit., p. 22.

${ }^{15}$ JAMES, Tim, op. cit., p. 25.

${ }^{16}$ Ibidem.
} 
volta, il ceppo ereticale valdese ${ }^{17}$. Il Duca, sotto pressione, alla fine decise di promulgare l' Editto con cui cancellava la libertà religiosa. Tale decisione ebbe come prima conseguenza lo spopolamento delle valli valdesi e sancì l'inizio dell'esodo Valdese verso i territori d'oltralpe, con Svizzera e Germania come mete privilegiate. Altri invece decisero di rifugiarsi presso le Province Unite Olandesi, sebbene i territori fossero già stracolmi di rifugiati.

Gli alti funzionari olandesi iniziarono ad elaborare una strategia che potesse risolvere il problema dell'accoglienza degli esuli: dirottare il flusso verso le colonie sembrò la soluzione ideale. Il Consiglio dei Diciassette esaminò le prospettive di tale proposta e decise di inviare famiglie ugonotte e successivamente, nell'inverno tra il 1687 e la primavera del 1688, famiglie valdesi rifugiate in Germania, a Norimberga, nei territori della Colonia del Capo come free burghers (liberi cittadini).

Il Consiglio dei Diciassette, inoltre, stabilì precise condizioni per gli emigrati: veniva garantito il trasporto gratuito sulle navi della Compagnia fino a Table Bay con conseguente dono di una somma di 125- 210 franchi ai capi famiglia e di 62-104 franchi agli emigranti celibi e concessione gratuita di terre da coltivare. Inoltre veniva anticipato dalla Compagnia del bestiame e degli attrezzi necessari ai lavori dei campi e garantita l'assistenza di un pastore di lingua francese. Oltre a ciò ai rifugiati valdesi venne riconosciuto lo status di correligionari ${ }^{18}$. A ciò seguiva l'obbligo di prestare giuramento in forma solenne:

Je promets et pure de restare fidale Leurs Hautes Puissances, le Etats Gènèraux des Provinces Unies, notre très haut et souverain gouvernement, à Son Altesse le prince d' Orange, gouverneur, capitaine et amiral général, au Directeur de la Compagnie générale à charte des Indes Orientales desdites Provinces, ainsi qu'au gouverneur gènèral et aux conseillers de l'Indie, enfin à tous les gouverneurs, commandeurs et officiers qui seront mes supérieurs sur mer et plus tard à terre. En toute occasion et de mon mieux j' observerai et ferai observer toutes le lois, dits et ordonnances publiés ou qui seront publiés par les Seigneur Directeurs, le Gouverneur gènèral et les conseillers de l'Inde ou le Gouverneur ou le Commandeur de ma future résidence, Enfin je promets en tous sens de me conduire ainsi qu'un bon et fidèle sujet est tenu de le faire. Ainsi fidèle, que Dieu Tout-Puissant, me sort en aide ${ }^{19}$.

Un giuramento che esplicitava le reali intenzioni del governo olandese che assunse un atteggiamento oscillante tra lo spirito umanitario di accoglienza e quello utilitaristico: i rifugiati

\footnotetext{
${ }^{17}$ RAINERO, Romain, «Il popolamento ugonotto della Colonia del Capo e le trattative per un emigrazione valdese nel Sud Africa attorno al 1688-89», in Bollettino della società degli studi valdesi, 116, 1961, pp. 33-42.

${ }^{18}$ BOTHA, Graham, The french Refugees at the Cape, Cape Town, C. Struyk (PTY) Ltd., 1970, p. 2.

${ }^{19}$ RAINERO, Romain, op. cit., p. 35, da ricordare come il giuramento venne tradotto dall'olandese al francese per agevolare la comprensione del testo. Il giuramento tradotto in italiano si trova in LUGAN, Bernard, op. cit., p.54.
} 
erano delle pedine, in mano olandese, facenti parte di un disegno molto più ampio in cui essi diventavano un investimento economico per la Colonia del Capo e per la Compagnia stessa. A conferma di ciò, Simon van Der Stel, appuntò in una lettere del 26 aprile del 1688 al Consiglio dei Diciassette le seguenti riflessioni: «Riteniamo di incontrare tra i rifugiati francesi ed i piemontesi che verranno, uomini esperti nella coltivazione della vite dell'ulivo che istruiranno i vecchi coloni che ignorano del tutto questa coltura» ${ }^{20}$.

La risolutezza politica del nuovo Governatore impresse un cambio deciso di rotta ai negoziati a cui fecero seguito i primi accordi e le prime partenze. Il Consiglio dei Diciassette si riunì nuovamente il 22 marzo del 1688 per esaminare una comunicazione del 19 Febbraio fatta dalla Camera di Amsterdam degli Stati Generali; in essa si leggeva:

Consta che una parte dei poveri profughi dalla valli del Piemonte ha trovato temporaneo rifugio nei pressi di Norimberga; si tratta di 200 famiglie che contano circa 1000 anime tra uomini, donne e fanciulli. essi sono esperti agricoltori ed in più muratori, carpentieri, ferraioli e bottai e tra costoro vi sono pure quatto pastori. Tutti sono inclini a partire per l'una o l'altra colonia sotto il dominio della Compagnia delle Indie orientali ed occidentali, alla sola condizione di essere autorizzati nei limiti del possibile ad essere sistemati gli uni vicino agli altri allo scopo di poter praticare assieme la loro religione per la gloria di Dio e per la sua futura estensione. Perciò le loro Alte Eminenze desiderano che i Diciassette dichiarino se possono o meno trovare una località conveniente ad un largo numero di questo povero popolo in uno o in un altro distretto della Compagnia ${ }^{21}$.

Venne intavolata una trattativa tra rappresentanti del governo olandese ed i rifugiati ugonotti e valdesi, il cui portavoce fu un tale Jean Pastre Marchand, commerciante che si recò personalmente ad Amsterdam. Le condizioni richieste dai rifugiati furono rese note dal Consiglio: invio a spese della Compagnia e degli Stati Generali di un primo contingente di 6-700 valdesi da assistere a lungo anche in altre colonie seguito dalla nomina di un comitato ristretto di tre persone incaricato di mantenere i contatti tra emigranti e governo e l' assicurazione per il Comitato della piena libertà di azione e di indagine in merito alla sistemazione dei valdesi.

Nel contempo iniziarono a montare polemiche e dubbi tra le fila valdesi; i capi-famiglia e gli esponenti di spicco della comunità ritenevano le condizioni proposte dagli olandesi lesive della libertà e della sopravvivenza della comunità. Iniziò così un periodo di intensi contatti tra le parti fin quando un episodio segnò la svolta: l'elettore del Brandeburgo avanzò una proposta di asilo con conseguente insediamento dei rifugiati valdesi presenti a Norimberga ${ }^{22}$. La maggioranza degli

\footnotetext{
${ }^{20}$ VON LEIBBRANDT, Hendrik Carel, Rambles through the Archives of the Colony of the Cape of Good Hope, 16881700, Capetown, J.C. Juta and Co., 1887, p. 37.

${ }^{21}$ Ibidem, p. 89.

${ }^{22}$ RAINERO, Romain, op. cit., p. 39.
} 
esuli valdesi, così, decise di non partire più ed aspettare in Germania che l'editto emanato dal Duca di Savoia venisse ritirato, per poi poter fare ritorno nelle proprie valli di origine ${ }^{23}$ nonostante fosse stata raggiunta una parziale intesa con gli olandesi: infatti venne redatto, il 1 aprile del 1688, un elenco di 126 persone tra Ugonotti e Piemontesi pronte ad imbarcarsi per la stessa destinazione dal porto di Amsterdam prima con la nave Schielandt e poi con la t' Wapen van Alkmaar. A seguito dello strappo tra comunità valdese ed autorità olandesi solamente pochi avventurieri tra questi, di lingua francese, con a seguito le proprie famiglie, decisero comunque di imbarcarsi per il Capo, facendosi passare per Ugonotti. Alcuni di loro arrivarono sulle coste del Sudafrica il 12 maggio del 1688, a bordo della Borssenburg ${ }^{24}$, partita il 6 gennaio dello stesso anno da Texel, prima che le trattative tra i valdesi e gli olandesi fossero naufragate. Tra di essi ricordiamo i vari Joubert, Malan, Jourdan, Davit, Durand, Fracha, Gardiol, Martinel, Peron, Pelanchon, Savoie, Malerba, Pisani, Lombard e Botta (Botha in Afrikaans) ed altri cognomi che al momento della registrazione da parte delle autorità olandesi vennero traslitterati e modificati o semplicemente francesizzati, così da rendere più difficile la distinzione tra ugonotti e valdesi ${ }^{25}$. Oltre a ciò subirono una ulteriore snazionalizzazione poiché furono obbligati ad integrarsi all'interno della società olandese del Capo, modificando ulteriormente i propri cognomi. Ad esempio il cognome Jourdan, tipico cognome di origine valdese, venne modificato in Jordaan e poi Jordan cognome presente ancora oggi nella regione del Capo (infatti è presente una winefarm chiamata Jordan Estate di cui però non è stato possibile, a seguito delle ricerche svolte in loco scoprirne le origini) ed ancora Pisani, venne francesizzato in du Pisanì o ancora Malerba, in Malerbhe e Lombardo in Lombard o Luumbard. Un processo di assimilazione ancora più evidente emerge se si consultano gli elenchi dei passeggeri imbarcati sulle navi olandesi e registrati all'arrivo sulle coste della colonia infatti molti cognomi hanno una triplice trascrizione che va dall'italiano al francese ed infine all'olandese ${ }^{26}$. Nelle liste complete dei rifugiati è possibile notare come accanto ad alcuni nomi non venga specificata la provenienza oppure il luogo di nascita dell'individuo mentre per altri soggetti, tutti ugonotti francesi, vengono riportate le generalità ed anche il successivo luogo d'insediamento. Ciò indurrebbe a pensare che coloro i quali non avevano dichiarato il luogo d'origine, ma

\footnotetext{
${ }^{23}$ I Diciassette scrissero al Capo: «these people, being averse to the sea and long voyage, had changed their minds and stelle in Germany, and that forty French Refugees bred to agricolture were being in sent out in 't Wapen van Alkmaar». Cit. in McCALL THEAL, George, The history of South Africa 1652-1795, London, G. Allen \& Unwin, 1915, p. 10. Nel 1689, quattro anni dopo la revoca dell'editto di Nantes, salì al trono Guglielmo III d'Orange il quale ricostituì il fronte anti-francese della Lega di Augusta ed inviò una spedizione ad agevolare il rimpatrio dei Valdesi nelle valli piemontesi. A capo della spedizione ci fu Herni Arnaud (16431721), che attraverso i valichi della Savoia dopo quattordici giorni sconfisse le truppe franco-sabaude a Salbertrand. Si compì così il Glorioso Rimpatrio (Glorieuse rentrée) dei valdesi nelle proprie terre d'origine. La piena libertà giunse soltanto con l'entrata in vigore dello Statuto Albertino nel 1848.

${ }^{24}$ Cfr. BOTHA, Graham, The French Refugees at the Cape, Cape Town, C. Struyk (PTY) Ltd. ,1970.

${ }^{25}$ RAINERO, Romain, op. cit., p. 40

${ }^{26}$ BOTHA, Graham, passim.
} 
solamente il proprio nominativo, fossero di origine valdese, avendo cognomi tipici delle valli piemontesi. Un esempio è quello di Jean Gardiol: nella lista completa dei rifugiati arrivati al Capo tra il 1688 ed il 1700 non si trovano dati inerenti al luogo di origine dell'individuo, ma solamente il nominativo. Facendo ulteriori ricerche ritroviamo il nome di Jean Gardiol riportato nel Bollettino della società degli studi valdesi, n.18, del $1900^{27}$, in cui, come si dirà tra breve, viene riportata la storia di un proprietario terriero, dal nome Jean Gardiol, nella zona di Stellenbosch. Questo tipo di ricerca incrociata potrebbe portare ad ulteriori risultati avvalorando la tesi secondo cui le autorità olandesi registrassero solamente i dati dei rifugiati francesi, molto meno difficili da capire e da trascrivere, per vicinanza geografica e conoscenza delle località francesi, rispetto a quelli dei valdesi. A ciò bisogna aggiungere il timore da parte delle autorità olandesi di concedere troppa autonomia ai rifugiati e ritrovarsi così delle enclaves autonome, di difficile governabilità, sul territorio della colonia. La soluzione a tale problema fu l'assimilazione e l'omologazione totale degli immigrati comprendente anche la modifica dei loro nomi e cognomi. Il nome in tale logica rappresentava un segno distintivo, un passaporto di origine da cancellare, eliminare, trasformando i nuovi arrivati in soggetti a-storici, privi di radici comunitarie e solidaristiche. Nonostante l'esiguità del materiale sulla comunità valdese del Capo è possibile affermare che l'arrivo dei nuovi coloni, sia essi valdesi sia ugonotti, segnò un passo decisivo ed importante per l'espansione della colonia e per l'incremento della produzione vinicola. I rifugiati ugonotti esperti nel campo agricolo e nella coltivazione della vite, importarono tecnologie e conoscenze fino ad allora sconosciute dai produttori del Capo. La produzione aumentò notevolmente, furono messi a coltivazione nuovi terreni ed il grosso della produzione si concentrò nella valle del Drakenstein, nel quartiere francese di Fransche Hoek, l'attuale Franschoek e nei pressi di Stellenbosch dove furono concentrati dopo il loro arrivo gli esuli e da qui ebbe inizio l'espansione del settore vitivinicolo sudafricano ${ }^{28}$.

Anche i valdesi, assimilati alla maggioranza ugonotta, fecero la loro parte, furono infatti i pionieri italiani (allora piemontesi) dell'emigrazione e della produzione vinicola in Sudafrica, come è testimoniato da alcuni documenti che riportano atti di concessione (venivano dati in concessione, ai migranti, terreni agricoli e farms per metterle in produzione ed iniziare un progetto di espansione coloniale pianificata) di fattorie e terreni. Alcuni esempi sono la Vrede in Lust, affidata a Jacques De Savoye, nella regione di Stellenbosch e sempre nella stessa area la Rhome et Languedoc data in concessione al piemontese Pietro Benozzi nel 1691 ed ancora La Cotte

\footnotetext{
${ }^{27}$ COCORDA, Oscar, «Les origines de la Colonia Hollandaise du Cap», in Bollettino della società degli studi valdesi, 18/1900, pp. 20-27.

${ }^{28}$ MONTBARBUT DU PLESSIS, Jean-Marie, Les Huguenots en Afrique du Sud, Montréal, L' Envers du décor-Les Editions Balzac, 2001.
} 
rilevata dal Jean Gardiol precedentemente citato ${ }^{29}$. Ad essi, nei secoli successivi succederanno altri italiani impegnati nel settore memori di un eredità passata importante ${ }^{30}$ di un know how utile all'innovazione ed alla creazione di nuove realtà economiche e sociali.

\section{L'arrivo degli inglesi tra ricerca della qualità e la sciagura della} fillossera

Il Governatore Simon Van der Stel (1679-1699) lasciò l'incarico nel 1699 al figlio Willem Adriaan il quale si dimostrò un uomo mosso da egoismo ed ambizione che frappose gli interessi privati a quelli collettivi. Sotto la sua guida nella colonia le tensioni tra coloni ed istituzioni aumentarono sfociando in aperto scontro quando il neo governatore cercò di accaparrarsi il monopolio della distribuzione del vino. A seguito di un'amministrazione poco oculata Willem Adriaan Van der Stel fu costretto a dimettersi ${ }^{31}$ nel 1707 e nel 1712 venne a mancare il padre, Simon, lasciando così la magnifica tenuta di Constantia priva di una vera guida e destinata allo smembramento. $\mathrm{Fu}$ il primo episodio in cui rifugiati ugonotti e valdesi si allearono e collaborarono con i burghers olandesi, sancendo una sorta di patto di solidarietà contro un nemico comune rappresentato dalla figura del Governatore tiranno. La proprietà della famiglia Van der Stel, centro, fino a quel momento, della sperimentazione agricola e vinicola, venne smembrata e divisa in Groot Constantia (Grande Constantia) e Klein Constantia (Piccola

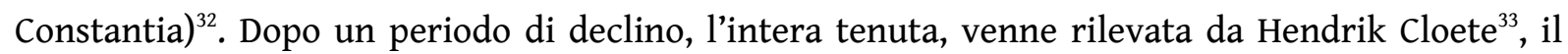
quale restituì lustro alla proprietà propiziandone la rinascita. Mise in produzione un vino che prese il nome di Constantia, un vero prodotto d'eccellenza, apprezzato e conosciuto finanche nei salotti dell'alta nobiltà europea: tra i suoi estimatori si annoverano Napoleone, Baudelaire, Federico di Prussia e molti alti dignitari dell'epoca ${ }^{34}$. Gli sconvolgimenti politici ed i venti rivoluzionari che spiravano dall'Europa sconvolsero il piccolo avamposto ai piedi del continente africano. A seguito dei cambiamenti degli assetti politici europei anche la colonia del Capo mutò pelle vivendo un periodo di confusione istituzionale in cui

\footnotetext{
${ }^{29}$ COCORDA, Oscar, op. cit., pp. 20-27; cfr. TROTTER, Alys F., «Old Cape Homesteads and their founders», in Cape Times, 25 dicembre 1898.

${ }^{30}$ Uno dei discendenti valdese più illustre nella storia del Sudafrica fu Pietro J. Joubert esponente di spicco, insieme a Paul Kruger, della Repubblica del Transvaal e generale boero nelle loro guerre contro gli inglesi. Perì durante il conflitto anglo-boero.

${ }^{31}$ Questo episodio viene citato da Gabriele Sani in SANI, Gabriele, op. cit., p. 15.

${ }^{32}$ JAMES, Tim, op. cit., p. 28.

${ }^{33}$ Hendrik Cloete era un proprietario terriero; possedeva la Nooitgedacht farm, nei pressi di Stellenbosch, quando nel 1778 rilevò la tenuta Constantia.

34 DALLA CIA, Giorgio, 300 anni di evoluzione vitivinicola ai limiti del continente africano, in Il contributo italiano alla diffusione della civiltà del vino nel mondo, Vicenza, Biblioteca Internazionale La Vigna, 2000, p. 91.
} 
inglesi ed olandesi si alternarono, fin quando nel 1814, il Congresso di Vienna sancì la definitiva assegnazione della colonia all'impero britannico ${ }^{35}$. Iniziò così un periodo di rinascita e di espansione per il settore vinicolo della colonia, che sfruttò i lasciti del periodo olandese e l'organizzazione delle winefarms (basti pensare ai grandi appezzamenti di terreno su cui erano impiantate le viti e le strutture delle farms: ricordiamo che sotto il Governatore Simon Van der Stel le cantine vennero risanate, ripulite e divennero veri e propri ambienti a se stanti, divisi dal resto della struttura principale della farm). Come scrive, Giorgio Dalla $\mathrm{Cia}^{36}$, produttore ed enologo italiano residente in Sudafrica: «é facile immaginare quindi che il governo inglese cercasse di incentivare l'importazione di vini dalla loro colonia del Capo a scapito della Francia, della Spagna e del Portogallo, aumentando le tariffe doganali. Nel 1859 il vino esportato in Inghilterra superò i 150 mila hl ${ }^{37}$.

Furono incrementati i terreni per la coltivazione, si cercò di migliorare la qualità del prodotto, vero punto debole che limitava l'esportazione e si cercò di stabilire una sorta di filiera produttiva censendo tutte le farms presenti sul territorio. Fu così che nel 1823 furono censite 374 winefarms e nel 1865 si contavano già 55 milioni di viti, un aumento esponenziale se considerate le giovani origini della viticoltura sudafrican ${ }^{38}$. Un periodo florido che conobbe un brusco arresto quando, alla fine dell'Ottocento e precisamente nel 1866, la fillossera ${ }^{39}$ distrusse i vitigni ed azzerò la produzione, rendendo vani tutti gli sforzi precedenti ${ }^{40}$. Da questo momento in poi si realizzò che per portare avanti una produzione di tipo industriale bisognava ripartire da zero con conoscenze e materiale umano ed artigianale di nuova generazione. In poche parole serviva modernizzare il settore, regolarizzarlo e renderlo meno vulnerabile.

In questi anni non si segnalano figure di spicco di origine italiana nel settore, è lecito pensare che i discendenti di quei Valdesi giunti al Capo quasi due secoli prima abbiano continuato ad occuparsi della cura dei vitigni ed abbiano concorso come i propri antenati all'espansione del settore.

\footnotetext{
${ }^{35}$ Cronologia degli eventi politici: $1795 \mathrm{i}$ britannici si insediarono e rimasero al governo fino al 1803, quando ritornano gli olandesi. Nel 1806 i territori del Capo tornarono in mano inglese e nel 1814 il Congresso di Vienna ne riconobbe definitivamente l'influenza britannica.

${ }^{36}$ Giorgio Dalla Cia, friulano di origini, trasferitosi in Sudafrica negli anni Settanta è uno dei produttori vinicoli più conosciuti e noti. Diplomato presso la Scuola enologica di Conegliano in Italia ha messo le sue conoscenze al servizio di diverse grandi aziende prima di fondare una sua distilleria a Stellenbosch.

${ }^{37}$ DALLA CIA, Giorgio, op. cit., p. 91.

${ }^{38}$ Ibidem; cfr. Report of the Select Committee on Government Wine Farm (1889), in JS Gericke Library, University of Stellenbosch (South Africa), loc. E PAM, 18 cap.

${ }^{39}$ La fillossera della vite (Daktulosphaira vitifoliae) è un insetto della famiglia dei Phylloxheridae.È un fitofago associato alle specie del genere Vitis che attacca le radici delle specie europee (Vitis Vinifera) e l'apparato aereo di quelle americane. Questo dannoso fitofago della vite, originario del Nordamerica, è comparso in Europa nella seconda metà dell'Ottocento e oggi è diffuso in tutti i paesi viticoli del mondo. Provoca in breve tempo gravi danni alle radici e la conseguente morte della pianta attaccata, con l'eccezione di alcuni vitigni americani.

${ }^{40}$ JAMES, Tim, op. cit., p. 32.
} 
Riguardo alla comunità Valdese, a seguito del processo di assimilazione descritto precedentemente, non è stato possibile reperire materiale documentario riguardante il XIX sec., se si eccettua, la presenza di Missionari come Giacomo Weitzecker e Luigi Adolfo Jalla ${ }^{41}$. Saranno i decenni successivi a segnare indelebilmente la storia degli italiani nel settore e la storia della stessa viticoltura sudafricana, la quale compirà un passo decisivo verso la modernità e la sua affermazione a livello mondiale.

\section{Il Novecento e la modernizzazione del settore}

Il mercato vinicolo sudafricano si affacciò alle soglie del XX secolo in piena crisi, a causa della fillossera che aveva distrutto i vitigni ed a seguito della depressione post guerra anglo-boera che aveva avuto ripercussioni anche sul settore. Furono istituite diverse Commissioni Governative con l'incarico di misurare lo stato di salute dell'industria vinicola e ricercare soluzioni al problema. Iniziarono ad essere impiantate viti innestate su un ceppo americano e venne promossa la formazione di cooperative basate sull'utilizzo di tecnologie avanzate così da abbattere i costi di produzione con maggiori profitti per tutti. A seguito dei finanziamenti governativi nacquero nove cooperative come la Drostdy di Tulbagh fondata nel $1906^{42}$. Tale politica impresse nuova linfa vitale al settore e soprattutto segnò una tappa fondamentale nella creazione di una "South African wine production" ${ }^{43}$, un settore dell'economia non solo della regione del Capo ma di tutto il territorio nazionale. I Governi sudafricani si impegnarono a livello legislativo introducendo leggi che regolassero il mercato della produzione e della vendita, calmierando i prezzi e garantendo maggior controllo su tutte le fasi di produzione. Nel 1924 la K.W.V. ( Ko-operatieve Wijnbouwers Vereeniging) nata dalla fusione tra i produttori del Capo e l'associazione dei commercianti, venne incaricata dal Governo Smuts con la legge Wine and Spirits Control Act N. 5 di fissare il prezzo minimo per il vino da distillazione dando inizio ad un processo di controllo legale sullintera produzione vinicola ${ }^{44}$. Nel corso dei decenni si susseguiranno ulteriori provvedimenti legislativi che avranno come scopo principale la regolamentazione del sistema produttivo sudafricano con l'intenzione di raggiungere standard qualitativi di assoluta eccellenza così da potersi affermare

\footnotetext{
${ }^{41}$ Importante è ricordare come Giacomo Weitzecker fosse un missionario valdese inviato dalla società Evangelica di Parigi con il compito, nel 1884, di evangelizzare i territori del Basutoland. Dopo di lui altri missionari valdesi percorsero i sentieri dell'Africa australe: ricordiamo Luigi Jalla e la moglie Maria Jalla. Tale episodio viene riportato in WEITZECKER, Giacomo, I Valdesi nell'Africa australe, Torino, UTET, 1906, p.2223.

${ }^{42}$ Vedi in Report of Committee Nominated by Western Province (1905), in JS Gericke Library, University of Stellenbosch (South Africa), loc. SP (G.30-1905), Government Publications.

${ }^{43}$ Vedi in Report of the Select Committee on Improvement of the Wine Industry, in JS Gericke Library, University of Stellenbosch (South Africa), loc. E PAM 18 cap.

${ }^{44}$ DALLA CIA, Giorgio, op. cit., p. 91.
} 
anche sul mercato internazionale entrando in competizione con i vitigni californiani, italiani e francesi.

Il progetto era quello di fornire un'adeguata istruzione ai futuri enologi ed ai futuri professionisti del settore: si iniziò ad insegnare (a Stellenbosch) viticoltura ed enologia nel 1887 fino a quando nel 1918 venne fondata la facoltà di Agraria presso l'Università di Stellenbosch. Il punto di riferimento della neonata facoltà era la Scuola tedesca di Geisenheim, sia per affinità religiose sia perché in Sudafrica i vini bianchi, soprattutto quelli tedeschi, erano diventati i più apprezzati. Molti docenti e studenti sudafricani soggiornavano per lunghi periodi in Germania, dedicandosi a tirocini di lavoro e corsi universitari che avrebbero poi permesso agli stessi di fregiarsi del titolo accademico tedesco, meglio spendibile e prestigioso di quello conseguito nella madrepatria. In tale contesto è bene ricordare come nel 1925 il professor Perold, dell'Università di Stellenbosch, incrociando il Pinot Nero ed il Cinsaut creò la varietà del Pinotage, ritenuta da tutti il primo vino veramente Sudafricano ${ }^{45}$.

Fino alla metà del Novecento le tecnologie ed i modi di produzione venivano mutuati dai maestri tedeschi, ignorando la tradizione francese e le tecnologie italiane, che avevano dato inizio all'industria viti-vinicola sudafricana e che saranno riutilizzate nei decenni successivi sotto la decisa spinta dei produttori italiani.

\section{Gli italiani e l'enologia sudafricana contemporanea}

Nel contesto di restaurazione del settore viti-vinicolo sudafricano si affermarono importanti esponenti dell'imprenditoria e del mondo accademico di origini italiane che risulteranno essere tra maggiori innovatori del comparto promuovendo una rivoluzione socio-culturale nella produzione e nel consumo del vino.

Il primo grande nome che si affermò nel panorama viti-vinicolo sudafricano fu Francesco Eschini, originario di Pontremoli, arrivato a Cape Town dall'Argentina con tutta la famiglia a cavallo tra Ottocento e Novecento ${ }^{46}$. Iniziò la sua attività come gestore di una pensione, che ospitava soprattutto connazionali. Stando a stretto contatto con essi imparò quelle che erano le esigenze primarie dei pensionanti. Molti richiedevano del vino da tavola da accompagnare ai pasti, non quello corposo e robusto che veniva prodotto nella zona del Capo. Eschini a tal proposito si rivolse ad un possidente locale, un certo D.J. Krige, proponendogli di produrre vini leggeri, per il consumo quotidiano. Dopo un'attenta analisi del progetto e delle competenze i due si accordarono e poterono iniziare la produzione. In breve tempo il nuovo tipo di vino si affermò a

\footnotetext{
${ }^{45}$ Estratto dall'intervista con Giorgio Dalla Cia, del 25 agosto 2015, Stellenbosch (Sudafrica).

${ }^{46}$ SANI, Gabriele, op. cit., pp. 188-189.
} 
livello nazionale fin tanto da costringere l'intraprendente Eschini ad aprire una cantina propria, a Bellville, dotandola di ampi locali con serbatoi di cemento ad alta capacità e tecnologie moderne. La Bellville Winery continuò la sua intensa produzione di vini da tavola fino a quando nel 1926 venne rilevata dalla ditta Luigi Fatti di Johannesburg, anche questa di chiare origini italiane.

Il toscano Eschini avviò la produzione di vini in un momento importante della storia enologica del paese, in piena fase di ricostruzione dopo le sciagure della fillossera ed in un contesto politicosociale avverso alla presenza di immigrati, sopratutto italiani. A lui va il merito di aver introdotto nel mercato del vino sudafricano un prodotto fino ad allora sconosciuto, il vino da pasto, che nel corso dei decenni entrerà prepotentemente nel costume enogastronomico locale ${ }^{47}$ iniziandone $\mathrm{a}$ modificare lentamente i lineamenti elitari che fino ad allora ne avevano caratterizzato lo sviluppo.

Anche il piemontese Michele Angiolo Zoccola, trasferitosi nel 1888 a Johannesburg dall'Inghilterra, aprì il Gran National Hotel ed acquistò una farm dal nome Bergvlei di 1600 ettari che ribattezzò Lombardy Estate (oggi Wynberg presso Sandton). Fu il primo ad impiantare le viti nel Transvaal, fino ad allora ritenuto un territorio aspro ed inadatto ad ospitare vitigni, affermandosi sul mercato sudafricano e divenendo una colonna portante della comunità di Johannesburg e consigliere della Witwatersrand Agricultural Society.

Un'altra figura di spicco del panorama enologico italo-sudafricano è sicuramente quella del Dottor Nino Costa, innovatore, precursore dei tempi che con la sua preparazione e la sua intraprendenza introdusse nuovi modi di produzione.

Alla fine degli anni trenta si trasferì a Paarl ed inaugurò con l'ausilio dell'enologo friulano Agostini una fase di sperimentazione che servì per

la messa a punto di un massiccio concentratore di mosti a freddo importato dall'Italia. Durante la Seconda Guerra Mondiale ebbero così l'opportunità, sfruttando le infrastrutture frigorifere del concentratore, di sperimentare la prima fermentazione a temperatura controllata $\left(10-15^{\circ} \mathrm{C}\right)$ dei vini bianchi [...] Nino Costa invece continuò nel suo ruolo di innovatore, adottando nel 1960 il primo impianto di pastorizzazione per bottiglie di vino, usando un pastorizzatore tedesco per birra. Tra il 1962 e il 1964 riuscì a mettere a punto un ciclo integrale per l'elaborazione dei vini in continuo: partendo dal taglio dei vini, passava alla chiarificazione, stabilizzazione, filtrazione, imbottigliamento, tutto in continuo [...] nel 1963 iniziò i primi esperimenti di fermentazione in bottiglia con il sistema classico ${ }^{48}$.

\footnotetext{
${ }^{47}$ Ibidem.

${ }^{48}$ DALLA CIA, Giorgio, op. cit., p. 97.
} 
Il Sudafrica conobbe anche l'affermazione dei fratelli Moni, colossi dell'agroalimentare. La storia della famiglia Moni è inestricabilmente intrecciata con lo sviluppo dell'industria agroalimentare sudafricana. Tre dei sei fratelli Moni emigrarono alla volta del Sudafrica alla fine dell'Ottocento a seguito della crisi economica che aveva investito l'Italia; giunti all'estrema punta del continente africano e dopo diverse occupazioni i fratelli decisero di associarsi e dedicarsi al commercio di specialità alimentari italiane. Negli anni riuscirono ad espandere il proprio mercato e fondarono diversi empori in varie città del Sudafrica; investirono capitali nella produzione della pasta prima con la ditta Moni Brothers Pty. Ltd. e poi nella fusione con la famiglia Fatti che sfociò nella United Macaroni Factories Ltd.

Al loro impegno nella produzione della pasta affiancarono anche quello nel settore vinicolo: a causa della prima guerra i prodotto importati scarseggiavano e così i fratelli decisero di comprare una cantina a Huguenot e produrre in proprio i vini per poi smerciarli negli empori di proprietà. La scelta ebbe esiti positivi e nel 1922 la cantina Moni venne registrata come compagnia privata (Moni's Winery) divenendo un modello aziendale efficiente e all'avanguardia, avendo annesse anche la distilleria. Negli anni trenta ormai il vino dei Moni si era affermato a livello nazionale, spaziando dalla produzione di spumanti, a vini più leggeri, finanche al marsala e diversi distillati come il brandy ed altri liquori.

Nel secondo dopoguerra le Cantine Moni fecero segnare successi importanti come il conseguimento della medaglia d'oro ai Rand Shows del 1948 e del 1952, grazie soprattutto all'innovazione scientifica e la ricerca promossa e finalizzata nei laboratori di Huguenot dal Dottor Nino Costa. Nei decenni successivi, la società dei Moni assorbì la ditta L. Fatti \& Co. e controllava 15 compagnie, 24 locali di distribuzione, 6 bottiglierie e diverse rivendite di vini e liquori. Ancora oggi la famiglia Moni rappresenta l'élite industriale del Paese nel campo dell'agroalimentare e punto di riferimento della storia degli italiani in $100^{49}$. Cresciuti in un momento critico per l'economia sudafricana, i Moni riuscirono a trovare nelle difficoltà causate dalla prima guerra mondiale la chiave del proprio successo, introducendo nella cultura del paese la produzione industriale di pasta e l'introduzione del marsala e del moscato, sconosciuti all'epoca. L'azienda ha posto le basi di quella che sarà tutta la futura industria agro-alimentare del dopo-guerra ed ancora oggi il marchio Moni rappresenta il più grande produttore alimentare del Paese. Nel ramo vinicolo produce vini da tavola, spumanti, vermouth bianco e rosso, sherry, gin e molti altri vini ma ciò che resta dell'impegno dei fratelli Moni non è soltanto un colosso industriale ma il merito di aver contribuito, insieme agli altri produttori italiani del periodo, a modificare le abitudini enologiche sudafricane introducendo un prodotto italiano come il vino da

\footnotetext{
${ }^{49}$ La storia dei Fratelli Moni viene descritta nei testi SANI, Gabriele, op. cit., pp. 193-195; DALLA CIA, Giorgio, op. cit., p. 97.
} 
pasto ed iniziando un processo di democratizzazione dei consumi che travalicava le frontiere del colore e della razza. ${ }^{50}$

\section{L'ultima generazione di produttori italiani}

Il presente paragrafo è il frutto della ricerca sul campo in Sudafrica, nella zona di StellenboschPaarl, centro per eccellenza della produzione vinicola del Paese. L'esperienza diretta ed il contatto con la realtà dei produttori di vino ed i farmieri italiani ha permesso una maggiore comprensione del fenomeno qui trattato e soprattutto è stata utile nella fase di ricostruzione storica dell'intero settore enologico. Le testimonianze, le ricostruzioni, la semplice interazione e la partecipazione diretta ad un evento, ad una fase produttiva, quale può essere la distillazione o l'imbottigliamento del vino, permettono al ricercatore di carpire, sentire, storie, aneddoti e racconti di esperienze personali che arricchiscono la ricerca d'archivio.

Gli anni Settanta hanno visto l'affermazione di Giorgio Dalla Cia, sicuramente uno dei personaggi più influenti nella storia enologica sudafricana e si colloca in quella schiera di italiani che nel settore sono riusciti ad innovare e ad aprire nuovi mondi enologici fino ad allora sconosciuti.

Nel 1976 entrò nel Gruppo Distillers Corporation, a seguito della chiusura degli stabilimenti della Stock, e si trasferì a Stellenbosch, uno dei maggiori centri enologici del Paese. Assunse l'incarico di dirigere le Cantine Meerlust, dove le sue idee e le sue proposte vennero da subito accettate dal proprietario Nico Myburgh che lo spronò a mettere in produzione dei vini di tipo bordolese, un prodotto poco conosciuto ed apprezzato ai tempi. Nel corso degli anni, Giorgio Dalla Cia, mise appunto un vino di tipo Medòc e mutò radicalmente tutta la tecnologia e le modalità produttive precedenti (di stampo tedesco, come precedentemente illustrato) sostituendoli con la tradizione francese. Lo stesso Dalla Cia dice:

Quando sono arrivato possedevo delle tecnologie abbastanza avanzate che qui non si conoscevano; ho, in un certo qual modo, reinventato la tecnologia della fermentazione dei vini rossi ed anche dello Chardonnay. Infatti il mio vino è stato considerato ed ancora lo è, tra i migliori vini bordolesi nel mondo ${ }^{51}$.

\footnotetext{
${ }^{50} \mathrm{~A}$ tal proposito servirebbe un ulteriore approfondimento che tenga conto dell'impatto socio-culturale dell'industria vinicola sudafricana sulla società e sulle relazioni comunitarie in quanto per molti secoli promotrice di disuguaglianze e fratture sociali. Questa però è un'altra storia che si discosta dal focus del presente lavoro e per il quale servirebbe maggiore approfondimento ed una trattazione autonoma. Sul tema si rimanda a FOURIE, Johan, VON FINTEL, Dieter, passim.

${ }^{51}$ Estratto dall'intervista con Giorgio Dalla Cia, del 2 settembre 2015, Stellenbosch (Sudafrica).
} 
Come Cesare che pronunciò le fatidiche parole sul Rubicone "Alea Iacta Est», il primo vino prodotto seguendo il metodo bordolese, chiamato appunto Rubicon, con accentuato valore metaforico, segnò l'inizio di una nuova era per il settore enologico sudafricano e la conquista, non di Roma, ma dei mercati nazionali ed internazionali. Nel 1980 il Rubicon fu premiato, in Sudafrica, come miglior vino ed il Dalla Cia stesso come miglior produttore e nel 1984 produsse il Merlot, oggigiorno uno dei vini più apprezzati e presenti a livello mondiale. Come lui stesso dice in uno stralcio d'intervista: «Il Merlot è uno o forse il vino più amato dai sudafricani e viene apprezzato nell'intero Paese ed in tutto il mondo» ${ }^{52}$ puntellando quel processo di cambiamento nel consumo e nelle abitudini dei sudafricani.

Facendo tesoro dell'esperienza accumulata nei decenni precedenti e della fama riconosciutagli, alla metà degli anni Novanta, Giorgio Dalla Cia aprì una distilleria propria e riuscì, dopo vari tentativi, a produrre due vini, il Pinot Nero Riserva, raffinato in barrique di Allier, e lo Chardonnay, anch'esso fermentato in barrique di Allier. Da ultimo mise in produzione la grappa di Pinot-Chardonnay e quella di Cabernet-Merlot.

Un altro affermatissimo produttore di vini è Giulio Bertrand ${ }^{53}$, industriale toscano del settore laniero, che nel 1992 decise di ritirarsi in Sudafrica dall'Italia in una tenuta storica. Comprò Morgenster, fondata nel 1711, con l'intento di ritirarsi a vita privata ma nel contempo comprese le potenzialità del territorio e del mercato in espansione del Sudafrica decidendo con forza di piantare delle viti. Da ciò nacquero due vini rossi di stile Bordeaux di fama internazionale: il Morgenster ed il Loures River Valley che hanno ricevuto negli anni prestigiosi riconoscimenti. Dall'intervista del Dalla Cia:

Sulle orme paterne nel momento del ritiro dall'attività Giulio liquidò l'azienda in Italia, era un industriale del comparto tessile, e decise di investire qui in Sudafrica, restaurando Morgenster, un'antica farm. Iniziò a mettere a coltura gli ulivi, importando dall'Italia le varietà che non si trovavano in Sudafrica. Divenne così il più grande produttore di olio della nazione. Ha vinto, in Italia, per dodici anni consecutivi il premio l'Orciolo d'Oro per il suo olio extravergine. Così pian piano iniziando dall'olio decise di piantare viti: lo portai a Bordeaux per un soggiorno di tre settimane nel quale visitammo tutte le Chateau, tra cui ricordo la Chateau Cheval Blanc. Tornato dal viaggio chiese la mia consulenza per aprire una cantina che oggi è affermata e conosciuta in tutto il Paese ${ }^{54}$.

L'ultima generazione, cronologicamente parlando, di produttori vinicoli e farmieri italiani è rappresentata da una coppia di giovani friulani, Michela e Vittorio Dal Piaz: nel 2004 rilevarono la

\footnotetext{
${ }^{52}$ Ibidem.

${ }^{53}$ Nel periodo di permanenza in Sudafrica non fu possibile intervistare Giulio Bertrand a causa delle condizioni precarie di salute.

${ }^{54}$ Estratto dall'intervento del 13 settembre 2015 di Giorgio Dalla Cia, presso Ayama Farm (Paarl-Sudafrica), durante l'intervista a Michela Dal Piaz.
} 
Slendt Farms, una proprietà di 172 ettari, ai quali negli anni successivi si aggiunsero altri 48 ettari di terreno destinati alla produzione di vino.

Esperti del settore, valutarono subito le potenzialità del posto, che all'epoca produceva vini quali lo Chenin, il Cabernèt, lo Shiraz ed il Merlot e decisero di imprimere una svolta alla conduzione dell'azienda e diversificare il prodotto. Nel corso degli anni hanno sperimentato, hanno cercato e creato nuove "strade enologiche" arrivando a produrre dell'ottimo Pinotage, tra i migliori vini del Sudafrica fino all'esportazione intercontinentale del proprio prodotto.

Nel 2014, hanno conseguito un ulteriore primato, riuscendo a piantare la prima vigna di Vermentino (della Gallura) in Sudafrica, così da mettere in produzione uno dei pochi vitigni italiani, se si eccettua il Sangiovese, presente sul territorio nazionale:

Chiedemmo ad uno dei più importanti esperti di selezione clonale italiano nonché nostro amico, Augusto Fabbro, quale vitigno italiano avremmo potuto impiantare qui, in Sudafrica, così da avere qualcosa di nostro. Qui ci sono alcune varietà di rosso italiano ma di bianco non ne trovavi. Lui subito disse che questa era una zona per il Vermentino, specificatamente quello della Gallura, perché abbiamo terreni sabbiosi da sgretolamento granitico e vento caldo. Decidemmo di provarci ed importammo le viti: ci abbiamo messo sei anni per averle e finalmente nel novembre del 2014 abbiamo impiantato la prima vigna di Vermentino in Sudafrica ${ }^{55}$.

Una storia di successo che pone i proprietari di Ayama (il nome dell'azienda) nel solco della tradizione italiana del settore viti-vinicolo Sudafricano. Successo non solo in ambito produttivo ma anche dal punto di vista dell'integrazione e dell'adattamento culturale a contesti umani ed ambientali differenti:

Rilevare una proprietà terriera in Sud Africa non significa solo comprare del terreno per poi produrci, significa prendere con sé anche gli operai che ci lavoravano in precedenza, con tutti i problemi e le esigenze ad essi connessi. Significa vivere in simbiosi con loro, capirne le difficoltà, cercare di aiutarli: il proprietario terriero è un mentore, un punto di riferimento per la piccola comunità degli operai. Se c'è qualche problema vengono da noi a chiedere consiglio, a farsi aiutare. Essere proprietari è molto più complesso di quanto si possa immaginare ${ }^{56}$.

Soprattutto essere proprietari in un contesto culturale ed ambientale differente da quello di origine richiede adattamento e comprensione dei meccanismi socio-culturali vigenti. Come ricorda Michela dal Piaz nell'intervista:

\footnotetext{
${ }^{55}$ Estratto dall'intervista del 13 settembre 2015 a Michela Dal Piaz, presso Ayama Farm (Paarl-Sudafrica).

${ }^{56}$ Ibidem.
} 
Qui esiste ancora la Lobola, una sorta di prezzo della sposa o dote a cui devono far fronte le famiglie delle spose che intendono prendere marito. Quando alcuni non possono vengono a chiedere aiuto a noi, a chiedere consigli. Esistono ancora i riti di passaggio per diventare adulti: molti ragazzi al momento del passaggio tra adolescenza e maturità partono e vanno nella savana per qualche mese, praticano la circoncisione ed a volte non tornano. Noi dobbiamo conoscere e convivere con le realtà culturali del posto che sono importanti anche per il lavoro stesso nella farm e per la convivenza ${ }^{57}$.

In questo i giovani proprietari friulani hanno conseguito un ulteriore successo, riuscendo a costruire un rapporto simbiotico con i propri operai, facendosi carico di progetti di istruzione per i bambini, di dopo-scuola e di avviamento al lavoro.

Un'innovazione per il contesto sudafricano, che apre nuovi scenari per la produzione e la gestione di una farm ma anche per l'integrazione culturale requisito fondamentale per il successo di un'attività come quella descritta. Il concetto alla base di questa esperienza è ben descritto e rappresentato dal nome scelto per l'azienda: Ayama, una parola xhosa, dal significato importante ed evocativo, «qualcuno su cui contare».

Una storia significativa, importante sia dal punto di vista economico e sia dal punto di vista umano in cui l'osmosi tra il tradizionale concetto di proprietario terriero (farmer) e le esigenze della contemporaneità hanno inaugurato un nuovo cammino dell'industria viti-vinicola sudafricana ritenuta per molti secoli, ed a ragione, produttrice di ineguaglianze ${ }^{58}$ e non di congruenze.

\section{Conclusioni}

La storia del vino sudafricano è intimamente intrecciata alla storia della presenza italiana in Sudafrica e parlare di essa ci permette di descrivere come il contributo che le maestranze e le professionalità importate da questi ultimi abbiano avuto il merito di aver plasmato, modificato e spinto verso nuovi orizzonti, nuovi mercati e nuove dinamiche produttive il settore viti-vinicolo locale. Oltre a ciò, questa vicenda ha il merito di accendere i riflettori su una storia, quella dei migranti italiani del Sudafrica, poco studiata e relegata ancora oggi dagli specialisti ad un'analisi superficiale e priva di interesse.

\footnotetext{
${ }^{57}$ Ibidem.

${ }^{58} \mathrm{Cfr}$. FOURIE, Johan, VON FINTEL, Dieter, passim.
} 


\section{L'AUTORE}

Claudio SESSA è dottorando (Ph.D.) in Storia Contemporanea presso l'Università di Genova. Ha conseguito la laurea triennale in Scienze umane- antropologia presso l'Università di Siena e la laurea magistrale in Scienze Storiche presso l'Università di Bologna. È stato Affiliate Researcher tra l'agosto e l'ottobre 2015 presso l'Universiteit Stellenbosch (Sudafrica). Si è occupato di storia delle migrazioni ed in particolare di quelle italiane in Sudafrica, tema a cui ha dedicato le ricerche degli ultimi anni e su cui verte la sua tesi magistrale: Da ospiti indesiderati ad imprenditori affermanti: la migrazione italiana in Sudafrica e lo sviluppo del settore viti-vinicolo (1688-1970). Attualmente si occupa di storia economica del Corno d'Africa in particolare del passaggio storico-politico tra età pre-coloniale ed età imperiale.

URL: < http://www.studistorici.com/progett/autori/\#Sessa $>$ 